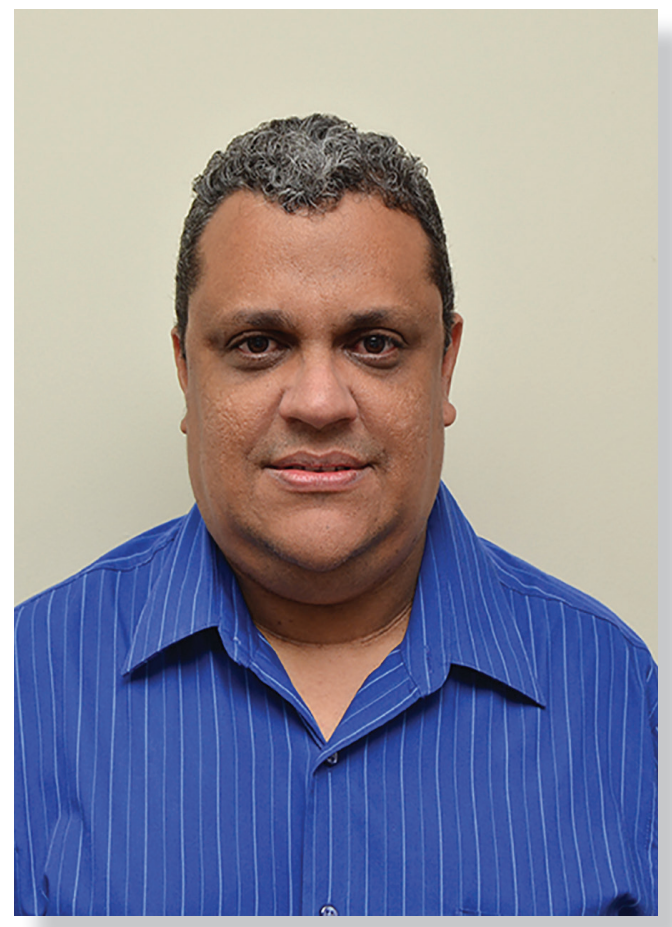

José Alejandro Rodríguez Núñez

\title{
Competencias comunicativas y su incidencia en el desarrollo de los procesos de comprensión y producción discursiva-académica
}

\section{Communication skills and their impact on the understanding and development of discourse- academic production}

Recibido: 04-12-15

Aprobado: 25-02-16

\section{Resumen}

Elpresenteartículotienelafinalidad devalorarlascompetencias comunicativas al servicio del uso de la lengua en contextos académicos. Destaca las cuatro capacidades específicas que pone de manifiesto el uso lingüístico: hablar, escuchar, leer y escribir; capacidades indispensables para la interacción social, en las que convergen habilidades lingüísticas, pragmáticas y discursivas.

Este artículo pretende demostrar la pertinencia de las competencias comunicativas a favor del desarrollo académico e integral del educando. Todo ello se traduce en habilidades cognitivas, procedimentales y discursivas.

\section{Abstract}

This article assesses the value of communication skills in academic contexts. It highlights four specific capabilities that showcase the use of linguistics: speaking, listening, reading and writing: all essential skills for social interaction that witness the convergence of linguistic, pragmatic, and discourse skills.

This article demonstrates the relevance of communication skills in students' holistic and academic development. It links the development of communication skills to overarching cognitive, procedural and discourse skills.

\section{Palabras clave}

Competencias- competencias comunicativas- competencias lingüísticas- procesos de comprensión y producción oral y escrita.

\section{Keywords}

Linguistic competencies, communication skills, learning processes, oral and written production

\footnotetext{
*José Alejandro Rodríguez Núñez: Licenciado en Educación, mención Letras por la Universidad Católica de Santo Domingo (UCSD); Postgrado y Maestría en Lingüística Aplicada por el Instituto Tecnológico de Santo Domingo (INTEC). Cursa actualmente el Doctorado en Estudios del Español: Lingüística y Literatura en la PUCMM y es profesor a tiempo completo y director del Departamento de Español, Campus Santo Tomás de Aquino. Para contactar al autor: joserodriguezn@pucmm.edu.do
} 
Las capacidades comunicativas, tales como hablar, escuchar, leer y escribir, evidencian, de algún modo, usos concretos de la lengua en contextos y ámbitos específicos; usos que se traducen en emisión o transmisión de mensajes (hablary escribir), y, a su vez, en recepción o incorporación de ideas. Estas capacidades implican un proceso interactivo y comunicativo de carácter social que experimenta todo sujeto en contexto, y que responde a una situación o circunstancia particular, con una intención o propósito que conlleva, a su vez, la necesidad de transmitir o recibir información.

En otras palabras, queda de manifiesto una situación comunicativa, cuyo eje central establece un vínculo entre los interlocutores, yo-tú; relación que, a juicio de Moquete (1999), hace de la lengua un modelo no solo lingüístico, sino además extralingüístico, en el que en forma discursiva se establece la ligazón entre lengua y cultura. Es así como la lengua se materializa en sistema o modelo basado en niveles de análisis de carácter social y funcional, que permite al sujeto hablante producir y recibir mensajes cuando este participa y actúa en una situación de comunicación que se concreta en discurso o texto oral o escrito.

Lo expuesto anteriormente constituye una situación cotidiana, sin excepción, pues día tras día, hora tras hora y minuto a minuto, experimentamos la necesidad de comunicarnos. El acto comunicativo es vital para la interacción social. En ese sentido, van Dijk (2000) sostiene que los usuarios del lenguaje utilizan activamente la composición textual y el habla no solo como hablantes, escritores, oyentes o lectores, sino además como miembros de categorías sociales, grupos, profesiones, organizaciones, comunidades, sociedades o culturas. Sin embargo, no se pretende en este artículo abarcar todas las esferas, reglas y dimensiones comunicativas, dada la diversidad textual y discursiva plasmada en los distintos géneros y tipologías textuales. Lo que sí se busca es situar el acto de comunicación en un ámbito o contexto específico: el académico. Este acto comunicativo estará basado, fundamentalmente, en el desarrollo de procesos de comprensión y producción, procesos inherentes a todas las disciplinas del saber, y que demandan, por lo tanto, ciertas habilidades cognitivas, lingüísticas y pragmáticas.

El desarrollo de capacidades potenciales y prácticas abarcan todo acto comunicativo. Dichas capacidades conllevan, además, una serie de destrezas, que, gracias a un sistema de conocimientos, procedimientos y actitudes, favorecen una comunicación eficaz. Es a lo que Molina (2006) llama competencias comunicativas.
En términos generales, y como bien plantea el Ministerio de Educación en República Dominicana (2014), las competencias comunicativas constituyen la base fundamental para el desarrollo humano, ya que posibilitan el contacto comunicativo a través de variados sistemas lingüísticos y no lingüísticos.

Estas competencias permiten la expresión de ideas, emociones, sentimientos y valores. Su desarrollo implica, por parte del sujeto, el dominio progresivo de las características y condiciones de distintas situaciones o circunstancias comunicativas inherentes a intenciones o propósitos de comunicación, roles asumidos como interlocutor (yo-tú), así como la asimilación de las características del contexto en que se produce la comunicación.

Cabe destacar, entonces, que en lo adelante habremos de apostar a una orientación didáctico-pedagógica funcional, basada en el enfoque pragmático, que favorezca el desarrollo de habilidades dialógicas continuas entre los actores principales del acto comunicativo (yo-tú), en ámbitos o contextos diversos y situaciones o circunstancias específicas: comprensión y producción oral y escrita. En este sentido, si bien es cierto que el saber lingüístico y comunicativo interactúan simultáneamente, hemos de favorecer el último; pues el saber lingüístico representa un recurso formal, al servicio de la necesidad comunicativa del sujeto en contexto.

Según Lomas (1999), si la enseñanza de las lenguas debe orientarse a favorecer el aprendizaje de las habilidades comunicativas, no basta con el aprendizaje de un saber lingüístico, entiéndase gramatical, que por sí solo no garantiza la mejora de las capacidades de uso expresivo y comprensivo. En efecto, el saber lingüístico debe convertirse en un aliado o recurso que contribuya a mejorar las competencias comunicativas en las diversas situaciones y contextos de comunicación. Es por ello que las competencias comunicativas representan el eje desde el que conviene articular la formación lingüística al servicio del saber decir (codificar) y el saber intepretar (decodificar) la información, como sostiene Lomas (1999).

Un perfil comunicativo adecuado debe responder satisfactoriamente a los diferentes contextos y situaciones comunicativas. Por esto se requiere, en adición al componente lingüístico, una serie de habilidades discursivas y sociolingüísticas, cuyo dominio hará posible el uso adecuado y correcto de la lengua en sociedad. Cassany (2007) considera estas habilidades como la manifestación concreta que 
permite la comunicación entre los miembros de una comunidad, cuyo signo de adscripción social establece la pertenencia a un grupo humano determinado.

Es así como las competencias comunicativas manifiestan una implicación social que va más allá del conocimiento lingüístico en sí mismo y de sus reglas. Estas competencias incluyen una serie de realidades vinculadas a hechos, acontecimientos y fenómenos de carácter social, destinados al establecimiento de la lengua como sistema convencional de signos empleados para la comunicación y la relación interpersonal y social en contextos determinados.

Proponemos, en adelante, un enfoque comunicativo que favorezca componentes actitudinales y un uso de la lengua más consciente, que integre los conocimientos lingüísticos y comunicativos, puestos al servicio del qué decir, cómo, cuándo y dónde (emisión), sin dejar a un lado quién lo dice, por qué y para qué, cómo, cuándo y dónde lo dice (recepción).

En definitiva, este enfoque se caracteriza, entre otros aspectos, por el perfil interdisciplinar que subyace en el uso concreto de la lengua, a través del texto; $y$, a su vez, por la dimensión interactiva puesta de manifiesto entre el sujeto (quién), el texto (qué) y el contexto (dónde, cuándo). Lo propuesto planteado hasta el momento será canalizado a continuación en cuatro dimensiones comunicativas específicas, que en suma ponen de manifiesto las competencias comunicativas.

\section{Comprensión oral}

Los procesos de comprensión oral están asociados a la recepción de la información, a través de la habilidad comunicativa denominada escucha. Esta habilidad, ajuicio de Cassany (2007), es la que suele despertar menos interés en la cotidianidad, ya que tiende a confundirse con la actitud pasiva y silenciosa del auditorio; pero que, en definitiva, implica comprender el mensaje. Para ello, debemos poner en marcha un proceso cognitivo de construcción e interpretación de significado, que exige unos procedimientos (reconocer, seleccionar, interpretar, inferir, anticipar); unos conceptos (adecuación, coherencia, cohesión, gramática, presentación) y unas actitudes (cultura oral, diálogo, receptividad, atención).

Vemos, entonces, que la capacidad de escucha, inherente a la comprensión oral, demanda ciertas habilidades diferenciadas, en parte, conforme a la modalidad discursiva, objeto de escucha. No será lo mismo escuchar un discurso espontáneo, en el cual la competencia del hablante es mínima, que escuchar un discurso controlado, con un nivel más alto de planificación por parte del hablante. En este caso, las capacidades inherentes a la competencia textual son similares a las de la lectura, mientras que, en el caso de la espontaneidad discursiva, el componente pragmático merece mayor consideración, ya que el texto producido demanda una referencia a la situación.

Para concretar estas habilidades, vinculadas a la comprensión oral, destacaremos cuatro subcomponentes involucrados en el proceso propuestos por Boeglin (2008), independientemente del discurso o texto objeto de escucha: subcomponente técnico, subcomponente semántico, subcomponente sintácticotextual y subcomponente pragmático.

El subcomponente técnico concierne a los aspectos exteriores, físicos, del código. Coincide con el componente fonológico, es decir, con la capacidad de identificar y reconocer los sonidos. La ausencia de este componente es indicio, casi siempre, de una patología. Prestarle atención durante todo el proceso discursivo será considerada una capacidad para desarrollar continuamente. Este subcomponente se manifiesta siempre que el oyente reconoce e identifica los esquemas de entonación que subyacen a la actitud global del hablante: pregunta, orden, afirmación, exclamación... Asimismo, surge cuando el escucha reconoce e identifica esquemas de entonación de frases enunciativas, interrogativas, imperativas, etc.

El subcomponente semántico capta la relación entre significados y significantes, a través de la propia experiencia y modelos conceptuales adquiridos. En este subcomponente, el oyente, a partir de una parte de una secuencia verbal, sabe hacer previsiones acerca de lo que sigue. De igual manera, reconoce el significado de la entonación y de las pausas, así como el significado y la función global de un enunciado, sin dejar a un lado el significado de los elementos deícticos, con referencia al contexto no verbal.

El subcomponente sintáctico-textual asume las relaciones que se producen en el eje sintagmático dentro del enunciado, así como las relaciones que se dan a lo interno del texto. Este subcomponente se evidencia al ponerse en práctica el orden de las palabras, la concordancia y los índices para reconocer las relaciones funcionales que se producen en el interior del enunciado. Remite correctamente las informaciones a los individuos a los que el hablante desea remitirlas.

Reconstruye mentalmente la estructura sintáctica lineal a partir de un texto oral convencionalmente imperfecto: elimina redundancias, falsos puntos de partida, 
comprende las relaciones entre las distintas partes del enunciado.

Finalmente, el subcomponente pragmático relaciona las informaciones recibidas con las características de la situación comunicativa en la que ha sido producido el mensaje: reconoce la intención comunicativa del hablante, comprende las principales informaciones transmitidas explícitamente, distingue las ideas principales de las secundarias.

\section{Producción oral}

Por tradición, la producción oral, como dimensión comunicativa, ha sido sustituida por la producción escrita, seguida de la lectura. Hablar bien o hablar mejor no forma parte de la formación integral y disciplinar del sujeto, pues se da por entendido que, por el hecho de ser habilidades "naturales" o "espontáneas", no requieren de unos procedimientos y unos conceptos que coadyuven a la adquisición del buen decir.

Si bien el habla cotidiana es espontánea, y hasta cierto punto libre de procesos de planificación, habrá ocasiones en las que producir mensajes orales constituirá un verdadero reto intelectual para quienes ponen de manifiesto su mensaje ante un público selecto, en un escenario particular y con una intención y una postura específica.

La práctica de la expresión oral no es superior, pero tampoco inferior, a la expresión escrita. Por tanto, demanda unos procesos simultáneos que hasta cierto punto son interdependientes. Pensemos que, al proponer a nuestros estudiantes planificar una exposición, por ejemplo, ellos tendrán que redactar; luego, al exponer, tendrán que leer, analizar e interpretar lo leído, y esto se traduce a la expresividad oral.

Es así como ciertas microhabilidades han de ser consideradas en estos procesos expresivos, tales como la indagación, la selección, la organización y planificación de las ideas: procesos tan rigurosos en la oralidad como en la escritura.

No olvidemos que una adecuada producción de la oralidad ha de cumplir unas propiedades específicas: adecuación, es decir, que la exposición se adapte a las características de los receptores; precisión o exactitud de lo que se quiere expresar sin dar cabida a la ambigüedad o confusión; corrección, basada en aspectos de la normativa, desde la pronunciación hasta la adecuación semántica, sin excluir la adecuada construcción sintáctica y el uso con propiedad de las palabras.
Finalmente, está la concisión, propiedad que prescinde de frases superfluas, evitando la prolongación innecesaria de frases vacías o superficiales.

\section{Comprensión escrita}

Leer es un acto interpretativo que consiste en saber guiar el razonamiento hacia la construcción de una interpretación delmensaje escrito a partirdelainformación que proporciona el texto. La lectura implica iniciar una serie de razonamientos para controlar el progreso de esa interpretación, de manera que se puedan detectar posibles incomprensiones producidas durante su proceso.

Colomer (1996) establece que en todo acto de lectura debe tenerse en cuenta la interrelación de tres factores claves: el lector, el texto y el contexto. El lector aporta los conocimientos que posee en sentido amplio; es decir, todo lo que es y sabe sobre el mundo, así como todo lo que hace durante la lectura para entender el mensaje. El texto, por su parte, se refiere a la intención del autor, al contenido de lo que dice y a la forma en que ha organizado su mensaje. En cambio, el contexto comprende las condiciones de la lectura, tanto las que fija el propio lector como las derivadas del entorno social.

La relación entre estas tres variables influye enormemente en la comprensión del texto, que será posible siempre que armonicen el texto y el lector, cuya interacción incluye la influencia del conocimiento previo a la lectura, el dominio de microprocesos y procesos inferenciales en niveles inferiores del texto. Incluye, además, la capacidad de entender globalmente el texto, de interpretarlo más allá de su información escrita y la posibilidad de controlar la lectura que se realiza, sin dejar a un lado la interacción lector y contexto.

\section{Producción escrita}

La situación de escritura incluye contenidos diversos que requieren procesos de enseñanza y aprendizaje, orientados desde una perspectiva discursiva y didáctica. Cassany (2007) propone varios enfoques interdependientes que favorecen la secuencia que amerita la producción escrita en contextos académicos. De ellos, describiremos los dos que consideramos más pertinentes para lograr una producción escrita orientada a la construcción de saberes.

El primero que destacaremos es el enfoque procesual. Este está asociado al proceso de producción e incluye etapas sistemáticas de composición. Implica el 
desarrollo de procesos cognitivos, tales como generar ideas, formular objetivos, organizar ideas, revisar, evaluar. Asimismo, enfatiza la composición del texto a través de la búsqueda de la información, elaboración de esquemas, redacción, evaluación y revisión del mensaje. El énfasis de este modelo radica en que todo texto conlleva el desarrollo de procesos metacognitivos vinculados a la puesta en marcha de actividades diversas que contribuirán a un producto final, distinto y mejorado, diferente a la producción concebida como un producto definitivo y cerrado.

El segundo enfoque a destacar es el basado en el contenido. En este se trabaja la escritura interdisciplinar, asociada con procesos intertextuales con el uso de citas y referencias. Debe desarrollarse dentro de cada disciplina, fundamentalmente a través de comentarios, reseñas, esquemas y resúmenes deconsultas bibliográficas y otros materiales escritos.

En definitiva, este enfoque implica procesos cognitivos de nivel superior en el marco de la producción de textos académicos. Por consiguiente, apostamos a la implementación de prácticas escriturales que favorezcan el desarrollo de competencias comunicativas orientadas de los enfoques descritos.

\section{Conclusión}

El desarrollo de las competencias comunicativas es indispensable para garantizar la puesta en ejecución de cualquier área disciplinar correspondiente al mundo académico. Sin estas competencias sería imposible acceder al conocimiento, objeto de estudio, análisis y expansión.

Cualquier área o disciplina del saber ofrece un caudal de información que amerita el acceso a la misma, ya sea por medio de la lectura (comprensión escrita) o de la escucha (comprensión oral). Una vez asumida la información, entonces construimos los saberes. Para ello es necesario declarar tal información, bien sea a través del habla (producción oral) o de la escritura (producción escrita).

En definitiva, las competencias comunicativas vistas hasta el momento (comprensión y producción oral; comprensión y producción escrita) constituyen el eje central de todo proceso académico en sus distintas manifestaciones contextuales. Para ello, alguien (yo) tendrá que dirigirse a una segunda persona (tú). El éxito de toda intercomunicación dependerá, básicamente, de unas habilidades lingüísticas, sociolingüísticas y pragmáticas, inherentes a las competencias comunicativas.

Al inicio de este artículo nos propusimos valorar las competencias comunicativas al servicio del uso de la lengua en contextos académicos, así como demostrar la pertinencia de estas competencias a favor del desarrollo académico e integral del educando. Creemos que los procesos de enseñanza y de aprendizaje que nos conciernen, independientemente de un área o disciplina en particular, deberían adoptar un giro más comunicativo-participativo y menos repetitivoinformativo.

Es preciso que, desde el diseño de nuestros planes de estudio, hasta los programas de asignatura, se incorporen, ya sea explícita o implícitamente, procesos comunicativos encaminados a fortalecer el desarrollo comunicativo, y así contribuir a la formación de un sujeto capaz de recibir y emitir información con eficiencia y eficacia.

\section{Referencias}

Boeglin, Martha (2008). Leer y redactar en la universidad. Bogotá: Magisterio.

Cassany, Daniel. (2007). Enseñar lengua. Barcelona: Graó.

Colomer, Teresa (1996). Enseñar a leer, enseñar a comprender. Madrid: Pedagogía.

Chomsky, Noaam (1974). Estructuras sintácticas.

Dijk, Teun (2000). El discurso como interacción social. Barcelona: Gedisa.

García, Bartolo. (2006). Competencias comunicativas. Santo Domingo: Surco.

Ministerio de Educación (MINERD) (2014). Diseño Curricular. Santo Domingo, República Dominicana.

Lomas, Carlos. (2002). El aprendizaje de la comunicación en las aulas. Barcelona: Paidós.

Lomas, Carlos. (1999). Cómo enseñar a hacer cosas con las palabras. Barcelona: Paidós.

Moquete, Manuel (1999). La Cultura de la lengua. Santo Domingo: INTEC.

Pérez, Héctor. (2006). Comprensión y producción de textos educativos. Bogotá: Magisterio. 